\title{
Preditores de Letalidade Hospitalar em Pacientes com Insuficiência Cardíaca Avançada
}

\author{
Predictors of in-hospital lethality in patients with advanced heart failure
}

Adriana Lopes Latado, Luiz Carlos Santana Passos, Julio C. V. Braga, Alessandra Santos, Rodrigo Guedes, Simone S. Moura, Daniela Almeida Hospital Português e Universidade Federal da Bahia - Salvador, BA

\section{Objetivos}

Descrever as características clínicas e identificar potenciais fatores de risco para letalidade hospitalar em pacientes com insuficiência cardíaca descompensada, admitidos em unidade de terapia intensiva.

\section{Métodos}

Pacientes consecutivamente admitidos por insuficiência cardíaca descompensada numa unidade de terapia intensiva, de junho/2001 a dezembro/2003, foram selecionados e seguidos durante a internação. Características clínicas na admissão foram coletadas e avaliadas por meio de modelos de regressão logística múltipla como preditores de risco para letalidade hospitalar.

\section{Resultados}

Foram avaliados 299 pacientes, 54\% do sexo masculino, com média de idade de $69 \pm 13$ anos. Doença arterial coronariana foi a causa principal da falência cardíaca em $49 \%$ dos casos. Diabetes mellitus e hipertensão arterial sistêmica ocorreram em 37,5\% e $78 \%$ dos pacientes, respectivamente. Na admissão, 22\% dos pacientes apresentavam fibrilação atrial; $21,5 \%$, disfunção renal e $48 \%$ dos casos, anemia (16,5\% com anemia importante). Encontrou-se disfunção sistólica grave (fração de ejeção do ventrículo esquerdo <30\%) em 44\% dos pacientes. A letalidade hospitalar foi 17,4\%. Após análise multivariada, história prévia de acidente vascular encefálico (AVE), fibrilação atrial, insuficiência renal, idade >70 anos e hiponatremia foram independentemente associados com letalidade hospitalar.

\section{Conclusão}

Pacientes internados por insuficiência cardíaca descompensada em unidade intensiva apresentam letalidade hospitalar elevada. Neste estudo, variáveis da admissão puderam predizer letalidade hospitalar, como AVE prévio, fibrilação atrial, hiponatremia, insuficiência renal e idade $>70$ anos.

\section{Palavras-chave}

Insuficiência cardíaca, epidemiologia, letalidade hospitalar, preditores.

\section{Objectives}

Describe the clinical characteristics and identify potential risk factors for in-hospital lethality in patients with decompensated heart failure admitted to an intensive care unit.

\section{Methods}

Decompensated heart failure patients consecutively admitted to an intensive care unit between June 2001 and December 2003 were selected and followed during hospitalization until discharge or death. Clinical characteristics at admission were recorded and evaluated as independent risk predictors for in-hospital mortality by multiple logistic regression analysis.

\section{RESULTS}

A total of 299 patients ( $69 \pm 13$ years of age and $54 \%$ men) were enrolled. Coronary artery disease was the main cause of heart failure in $49 \%$ of the cases. Diabetes mellitus and systemic arterial hypertension occurred in $37.5 \%$ and $78 \%$ of the patients, respectively. At admission, $22 \%$ of them had atrial fibrillation, $21.5 \%$ had renal dysfunction, and $48 \%$ anemia (16.5\% with severe anemia). Severe systolic dysfunction (left ventricular ejection fraction $<30 \%$ ) affected $44 \%$ of the patients. In-hospital mortality was $17.4 \%$. After the multivariate analysis had been performed, previous history of stroke, atrial fibrillation, renal failure, age $>70$ years, and hyponatremia were independently associated with inhospital mortality.

\section{Conclusion}

Patients admitted to an intensive care unit due to decompensated heart failure have high in-hospital lethality. In this study, variables recorded at admission, such as previous stroke, atrial fibrillation, hyponatremia, renal failure, and age $>70$ years were predictors of in-hospital lethality.

\section{KEY WORDS}

Heart failure, epidemiology, in-hospital lethality, predictors.

Correspondência: Adriana Lopes Latado - Rua Rosa dos Ventos, 39/1002 - 40223-010 - Salvador, BA 
A insuficiência cardíaca é uma doença de elevada prevalência e grande impacto na morbidade e mortalidade em todo o mundo, especialmente nos idosos ${ }^{1}$. 0 prognóstico em longo prazo ainda é ruim, com sobrevida em 5 anos menor do que $50 \%{ }^{1,2}$. De acordo com - DATASUS, ocorreram 340.000 admissões por insuficiência cardíaca no Brasil em 2004, responsáveis por $28 \%$ de todas as hospitalizações por doenças cardiovasculares e $3 \%$ das causas totais ${ }^{3}$.

Estudos observacionais em que se avaliam pacientes admitidos devido à insuficiência cardíaca são, em geral, restritos a centros únicos ou baseados em pequenos registros. Manifestações clínicas distintas e diferentes marcadores prognósticos têm sido descritos entre os pacientes com formas graves de insuficiência cardíaca comparados com aqueles portadores de formas mais leves da doença. Por exemplo, a fração de ejeção do ventrículo esquerdo parece não afetar o prognóstico de pacientes com insuficiência cardíaca avançada, oposto ao que ocorre com indivíduos com grau leve a moderado da doença ${ }^{4,5}$.

É possível que os progressos na terapia médica e cirúrgica da insuficiência cardíaca ${ }^{6}$, bem como o envelhecimento populacional, contribuam para aumentar a taxa de internação de casos mais avançados da doença, os quais podem ainda ser agravados pela coexistência de outras doenças crônicas. 0 estudo de pacientes portadores de insuficiência cardíaca grave, como é o caso daqueles que são internados em unidades para tratamento intensivo, é necessário e consistente com a demanda atual.

No presente estudo objetiva-se descrever as características clínicas e identificar preditores de letalidade hospitalar em pacientes internados por insuficiência cardíaca descompensada numa unidade de terapia intensiva da cidade de Salvador, Bahia.

\section{Métodos}

Desenho do estudo e amostra - Trata-se de uma coorte prospectiva de pacientes admitidos consecutivamente na Unidade de Terapia Intensiva (UTI) de um hospital terciário de Salvador, Bahia, com insuficiência cardíaca descompensada, entre junho de 2001 e dezembro de 2003. O diagnóstico de insuficiência cardíaca baseouse numa história clínica sugestiva (piora de dispnéia ou dispnéia de repouso) e presença de sinais de congestão pulmonar ou edema periférico, conforme critérios estabelecidos $^{7}$. Sempre que necessário, os resultados de exames complementares (raio $\mathrm{X}$ do tórax, ecocardiograma) também foram utilizados para estabelecer o diagnóstico da síndrome.

Os pacientes deveriam apresentar pelo menos uma internação por descompensação de insuficiência cardíaca no último ano, para serem considerados como portadores de doença crônica. O tempo de acompanhamento correspondeu ao período total de internação, já que os pacientes foram acompanhados após a saída da UTI, até a alta hospitalar. Foram exclusos os pacientes com diagnóstico definitivo de infarto agudo do miocárdio com supradesnivelamento do segmento ST.

Fonte de dados - As informações foram obtidas em consulta aos prontuários médicos. Os dados também foram obtidos por entrevistas diretas com o paciente, sempre que possível, ou com algum acompanhante. Em caso de dúvidas, era realizada uma consulta adicional ao cardiologista da UTI ou ao médico assistente do paciente. 0 instrumento de coleta de dados foi um questionário especificamente criado para esta pesquisa, incluindo variáveis demográficas, clínicas, relacionadas a exames complementares e terapia utilizada, além de informações sobre a evolução clínica hospitalar até a alta ou óbito. Este projeto de pesquisa está de acordo com os princípios delineados na Declaração de Helsinki ${ }^{8}$ e foi aprovado pelo Comitê de Ética em Pesquisa do próprio hospital. Foi solicitado um aceite do termo de consentimento livre antes da inclusão dos pacientes no estudo.

Exames complementares - O eletrocardiograma (ECG) de 12 derivações e os resultados de testes laboratoriais usados para análise foram aqueles obtidos na admissão do paciente. O exame laboratorial incluiu dosagens séricas de eletrólitos, uréia, creatinina e glicose, além de hemograma completo. 296 (99\%) pacientes submeteramse a ecocardiograma durante a internação para avaliação das câmaras cardíacas e funções sistólica e diastólica do ventrículo esquerdo. Cineangiocoronariografia foi realizada de acordo com a indicação médica do serviço, assim como ocorreu com outros exames complementares.

Definição de variáveis - As etiologias da insuficiência cardíaca foram definidas $\operatorname{como}^{9,10}$ : a) cardiopatia isquêmica pela presença de infarto do miocárdio prévio, angina de peito, revascularização coronariana prévia por angioplastia percutânea ou cirurgia, ou coronariografia que demonstraram obstruções arteriais críticas em ramos epicárdicos; b) cardiopatia hipertensiva, definida como história de hipertensão arterial sistêmica de longa data sem adequado controle pressórico ou uso prolongado de anti-hipertensivos, associada à presença de hipertrofia miocárdica ao eletrocardiograma ou ecocardiograma; c) cardiopatia valvular, definida como uma história de doença valvular prévia ou com base em resultados de ecocardiograma; d) doença de Chagas, definida por testes sorológicos positivos; e) miocardiopatia idiopática, quando dilatação e disfunção ventriculares estivessem presentes, na ausência de outra causa evidente; f) miocardite viral pela presença de disfunção ventricular esquerda e cintilografia miocárdica com gálio-67 ou biópsia endomiocárdica, sugestivas de anormalidades inflamatórias transitórias. Comorbidades foram definidas por história clínica, uso de medicações específicas ou resultados de exames complementares.

Insuficiência renal aguda durante a internação foi definida como nível de creatinina sérica $\geq 1,4 \mathrm{mg} / \mathrm{dl}$ naqueles com níveis basais normais ou um aumento de 
pelo menos $0,5 \mathrm{mg} / \mathrm{dl}$ naqueles cuja creatinina sérica da admissão fosse maior do que $1,4 \mathrm{mg} / \mathrm{dl}$.

A fração de ejeção do ventrículo esquerdo foi medida pelo ecocardiograma, por meio da fórmula de Teichholz para registros no modo $\mathrm{M}$ ou da fórmula modificada de Simpson para as medidas dos diâmetros sistólico e diastólico finais do ventrículo esquerdo, no corte apical das duas câmaras. Os valores da fração de ejeção do ventrículo esquerdo foram registrados nos seguintes intervalos: $>55 \%$ ou função sistólica normal, $45-55 \%$ ou disfunção sistólica leve, 30-44\% ou disfunção sistólica moderada e $<30 \%$ ou disfunção sistólica grave. Os diâmetros do ventrículo esquerdo foram medidos nos cortes paraesternais e dilatação do ventrículo esquerdo foi considerada presente quando diâmetro diastólico final fosse $>56 \mathrm{~mm}$. Insuficiência cardíaca com função sistólica ventricular esquerda preservada foi definida por fração de ejeção do ventrículo esquerdo $\geq 45 \%$. A função diastólica foi avaliada pelas medidas da velocidade transmitral inicial (onda E) e tardia (onda $A$ ), razão E/A e pelo tempo de desaceleração da onda $E$, e classificada nos padrões: normal, alteração do relaxamento ventricular, pseudonormalização e padrão restritivo ${ }^{11}$.

Converteram-se variáveis contínuas em variáveis categóricas para fins de análise estatística e estratificadas deste modo: idade ( $>70$ e $\leq 70$ anos), creatinina sérica $(<1,8$ e $\geq 1,8 \mathrm{mg} / \mathrm{dl})$ e sódio ( $\mathrm{Na})$ sérico $(<136 \mathrm{e}$ $\geq 136 \mathrm{mEq} / \mathrm{l}$ ). A presença de anemia foi definida conforme o critério da Organização Mundial da Saúde (OMS) 12: hemoglobina $(\mathrm{Hb})<13,0 \mathrm{~g} / \mathrm{dl}$ para sexo masculino e $\mathrm{Hb}<12,0 \mathrm{~g} / \mathrm{dl}$ para sexo feminino. Para fins das análises bivariada e multivariada, consideramos como subgrupo de risco os pacientes com níveis mais baixos de $\mathrm{Hb}$, correspondentes ao percentil 20 da amostra $(\mathrm{Hb}$ $<11,0 \mathrm{~g} / \mathrm{dl}$ para o sexo masculino e $\mathrm{Hb}<10,0 \mathrm{~g} / \mathrm{dl}$ para o sexo feminino), denominados como portadores de 'anemia importante'. Isto decorreu do fato de que, sendo a presente amostra composta por pacientes criticamente doentes, esperava-se que a prevalência de anemia, pelo critério da OMS, fosse muito alta, o que dificultaria o surgimento de possíveis diferenças entre os grupos de anêmicos e não anêmicos, caso de fato elas existissem.

Análise estatística - Variáveis contínuas foram descritas como médias \pm desvio padrão e comparadas pelo teste $t$ de Student para amostras independentes. Descreveram-se variáveis categóricas como proporções e comparadas pelo teste do qui-quadrado ou teste exato de Fisher. Fizeram-se análises bivariadas entre variáveis clínico-laboratoriais e morte hospitalar para estimativas brutas de associação (risco relativo) e seus respectivos intervalos de confiança (IC) de 95\%. Realizou-se análise multivariada exploratória por modelos de regressão logística para a determinação de potenciais fatores de risco independentes de letalidade hospitalar. Variáveis de admissão foram selecionadas para o modelo logístico exploratório se houvessem associado com morte hospitalar a um nível de significância $<10 \%$ na análise bivariada. 0 nível de significância (valor p) para todas as demais associações foi definido como 5\%, para hipóteses bicaudais. O software SPSS versão 10 foi usado para as análises.

\section{Resultados}

Características clínicas e eletrocardiográficas - Foram avaliados 299 pacientes. As características clínicas e demográficas principais estão descritas na Tabela 1. Os pacientes eram idosos (idade média de $69 \pm 13$ anos) e a cardiopatia isquêmica foi a causa primária da insuficiência cardíaca em quase metade dos casos. Observou-se hipertensão arterial sistêmica em $78 \%$ deles, um passado de AVE em 15\%, fibrilação atrial em $22 \%$ e diabete em $37 \%$ dos pacientes. A maioria desses apresentava dispnéia de repouso na admissão, dos quais $9 \%$ tinham síndrome de baixo débito.

Diabetes mellitus foi mais freqüente nos pacientes com etiologia isquêmica. Em pacientes com menos de 60 anos, essa associação foi mais forte, com razão de prevalência para diabete igual a 3,6 (IC 95\% 1,9-6,9) ao se compararem pacientes isquêmicos e não isquêmicos. Indivíduos com

\begin{tabular}{|c|c|c|}
\hline & $\mathbf{N}$ & $\%$ \\
\hline $\begin{array}{l}\text { Idade (anos) } \\
\qquad \text { Média } \pm \text { DP } \\
>70\end{array}$ & $\begin{array}{c}69 \pm 13 \\
144\end{array}$ & 48,5 \\
\hline Sexo masculino & 163 & 54,5 \\
\hline $\begin{array}{l}\text { Etiologia } \\
\text { Isquêmica } \\
\text { Hipertensão arterial } \\
\text { Valvar } \\
\text { Doença de Chagas } \\
\text { Idiopática } \\
\text { Viral }\end{array}$ & $\begin{array}{c}147 \\
77 \\
35 \\
29 \\
9 \\
3\end{array}$ & $\begin{array}{c}49,2 \\
25,8 \\
11,7 \\
9,7 \\
3,0 \\
0,7\end{array}$ \\
\hline Doença arterial coronariana & 175 & 58,5 \\
\hline Hipertensão & 233 & 77,9 \\
\hline Diabetes mellitus & 112 & 37,5 \\
\hline AVE prévio & 46 & 15,4 \\
\hline Disfunção renal na admissão & 137 & 45,9 \\
\hline Tromboembolismo venoso prévio & 24 & 8,0 \\
\hline Classe funcional IV (NYHA) na admissão & 229 & 76,5 \\
\hline Fibrilação atrial* & 65 & 22,0 \\
\hline QRS $\geq 120$ mseg* & 135 & 47,4 \\
\hline $\begin{array}{c}\text { Anemia na admissão (OMS) } \\
\text { Anemia importante }^{\#}\end{array}$ & $\begin{array}{c}144 \\
49\end{array}$ & $\begin{array}{l}48,0 \\
16,5\end{array}$ \\
\hline Bloqueio completo do ramo esquerdo* & 62 & 22,0 \\
\hline $\begin{array}{l}\text { Disfunção Sistólica } \\
\text { Moderada } \\
\text { Grave }\end{array}$ & $\begin{array}{c}64 \\
131\end{array}$ & $\begin{array}{l}21,6 \\
44,2\end{array}$ \\
\hline $\begin{array}{l}\text { Insuficiência cardíaca com } \\
\text { função sistólica preservada }\end{array}$ & 101 & 34,1 \\
\hline Dilatação do ventrículo esquerdo & 223 & 76,4 \\
\hline \multicolumn{3}{|c|}{$\begin{array}{l}\text { NYHA - New York Heart Association; * Eletrocardiograma } \\
\text { na admissão; OMS = Organização Mundial da Saúde; \# } \\
\text { Hemog/obina }<10,0 \mathrm{~g} / \text { dl para mulheres e }<11,0 \mathrm{~g} / \text { dl para homens; } \\
\text { AVE - acidente vascular encefálico }\end{array}$} \\
\hline
\end{tabular}


fração de ejeção do ventrículo esquerdo $<30 \%$ tiveram complexos QRS mais largos (60\% versus 37\%; $p<0,001$ ) e freqüência maior de bloqueio completo do ramo esquerdo (32\% versus $19 \%$ ), quando comparados com pacientes que apresentavam fração de ejeção $\geq 30 \%$.

Resultados ecocardiográficos - Foi realizado ecocardiograma em 296 pacientes. Os principais resultados estão resumidos na Tabela 1 . Encontrou-se disfunção sistólica grave do ventrículo esquerdo em $44 \%$ dos casos. Ocorreu insuficiência cardíaca com função sistólica preservada em 34\% dos pacientes. Dos indivíduos com disfunção valvular, $80 \%$ exibiram regurgitação mitral moderada a grave.

Achados laboratoriais - Resultados laboratoriais anormais à admissão foram freqüentes. Anemia foi encontrada em 48\% da amostra total: $43 \%$ no sexo masculino e $53 \%$ no sexo feminino $(p=0,09)$. Anemia importante ocorreu em 16,5\% dos casos. Níveis anormais de potássio sérico ocorreram em $13 \%$ dos pacientes. Encontrou-se hiponatremia em 21\% dos casos, dos quais 34\% apresentavam níveis menores do que $130 \mathrm{mEq} / \mathrm{l}$. Níveis elevados de creatinina ocorreram em $21,5 \%$ (65 casos).

Eventos hospitalares - Detectou-se embolia pulmonar em $15 \%$, sepsis em $5,7 \%$ e AVE agudo em $4 \%$ dos casos durante a internação. Desenvolveu-se insuficiência renal aguda em $42 \%$ dos pacientes.

A letalidade hospitalar foi $17,4 \%$. Os pacientes que faleceram eram, em média, mais velhos do que os que sobreviveram à hospitalização $(73 \pm 12$ anos versus $69 \pm 13$ anos; $p=0,03$ ). Na análise bivariada, algumas variáveis foram associadas $(p<0,05)$ com maior risco de morte: AVE prévio; fibrilação atrial, creatinina elevada (*1,8mg/dl) e anemia importante à admissão; dilatação ventricular esquerda; disfunção valvular moderada a grave (80\% correspondiam à insuficiência mitral); insuficiência renal aguda e AVE durante a internação atual. Quando a idade foi analisada como variável categórica (>70 e $\leq$ 70 anos), observou-se uma associação estatisticamente significante entre idade avançada e óbito $(R R=2,0$; IC 95\% 1,2-3,5). Hiponatremia grave à admissão aumentou o risco de morte hospitalar, porém com nível de significância marginal ( $R R=2,0$; IC 95\% 1,1$3,9 ; p=0,07)$. O uso de aminas vasoativas durante a internação teve forte associação com letalidade $(R R=20,3 ;$ IC 95\% 9,0-45,7). Fração de ejeção do ventrículo esquerdo, gênero e etiologia isquêmica não tiveram associação estatisticamente significante com letalidade hospitalar nesta coorte de pacientes com insuficiência cardíaca avançada.

Análise multivariada - Os resultados da análise de regressão logística múltipla estão apresentados na Tabela 2 . Os potenciais preditores independentes de letalidade hospitalar, entre as variáveis colhidas na admissão, foram: história prévia de AVE (RR=2,55; IC 95\% 1,17-5,55), creatinina sérica $\geq 1,8 \mathrm{mg} / \mathrm{dl}(\mathrm{RR}=2,27$; IC 95\% 1,13$4,54)$, fibrilação atrial ( $R R=2,18$; IC 95\%1,09-4,36), idade $>70$ anos $(R R=1,92$; IC 95\%1,00-3,70) e hiponatremia ( $R R=3,06$; IC 95\%1,08-8,67). A presença de anemia importante, apesar de associada a maior risco de morte hospitalar $(R R=2,02)$ após o ajuste, não se confirmou com preditor independente de letalidade ao nível de significância estatística de 5\%.

\section{Discussão}

Este estudo, com pacientes com insuficiência cardíaca grave admitidos em unidade intensiva, demonstrou que a história prévia de AVE, disfunção renal e hiponatremia na admissão, idade $>70$ anos e fibrilação atrial em ECG inicial são potenciais preditores, independentemente de letalidade hospitalar. Observou-se uma alta prevalência do sexo feminino, de pacientes idosos e de comorbidades, como diabetes mellitus, hipertensão arterial sistêmica e insuficiência renal. Vale ressaltar que o estágio avançado da insuficiência cardíaca foi definido primariamente por critérios clínicos, associados à necessidade de internação em unidade intensiva.

Estudos que envolvem pacientes com insuficiência cardíaca grave são escassos, geralmente retrospectivos e baseados em amostras de indivíduos inclusos em programas para transplante cardíaco ${ }^{5,13}$. Pacientes selecionados para transplante, embora quase sempre com falência cardíaca terminal, são mais jovens e atendem a critérios de inclusão muito rígidos. Conseqüentemente, é possível que os resultados aqui apresentados representem mais adequadamente os pacientes com insuficiência cardíaca avançada, atendidos na prática clínica.

Caracterização dos pacientes - Como citado, a média de idade dos pacientes foi alta e houve um grande percentual do sexo feminino neste estudo. Trabalhos prévios mostraram que mulheres com insuficiência cardíaca são mais velhas do que os homens e

Tabela 2 - Análise Multivariada Exploratória para Letalidade Hospitalar

\begin{tabular}{|c|c|c|c|}
\hline Características da Admissão & Odds ratio & Intervalo de Confiança 95\% & Valor de $p$ \\
\hline AVE prévio $^{\prime}$ & 2,40 & $1,09-5,29$ & 0,03 \\
\hline Fibrilação Atrial & 2,35 & $1,17-4,72$ & 0,02 \\
\hline Hiponatremia $(\mathrm{Na}<130 \mathrm{mEq} / \mathrm{l})$ & 2,88 & $1,01-8,22$ & 0,05 \\
\hline Insuficiência Renal ( $C^{\ddagger} \geq 1,8$ mg/dl) & 2,27 & $1,13-4,54$ & 0,02 \\
\hline Idade $>70$ anos & 1,92 & $1,00-3,70$ & 0,05 \\
\hline Anemia importante* & 2,02 & $0,96-4,25$ & 0,06 \\
\hline
\end{tabular}


apresentam-se mais freqüentemente com função sistólica do ventrículo esquerdo preservada ${ }^{14-18}$. No presente estudo também encontrou-se uma elevada proporção ( $1 / 3$ dos casos) de insuficiência cardíaca com função sistólica preservada, o que provavelmente decorreu do perfil de nossa amostra. Neste aspecto, alguns estudos têm demonstrado resultados discordantes, geralmente devido ao emprego de diferentes critérios de inclusão para selecionar os pacientes com falência cardíaca. Por exemplo, no estudo EPICAL ${ }^{9}$ avaliou-se uma amostra com insuficiência cardíaca avançada que foi primariamente definida pela presença de disfunção sistólica grave do ventrículo esquerdo (fração de ejeção $<30 \%$ ), o que resultou na seleção de pacientes mais jovens e, na grande maioria (75\%), do sexo masculino. Ensaios clínicos randomizados em insuficiência cardíaca, por sua vez, também tendem a selecionar amostras com baixa freqüência do sexo feminino (em geral, inferior a 20\%) ${ }^{18}$ o que não é consistente com a realidade da prática clínica; a generalização de seus resultados fica, portanto, limitada para o subgrupo de mulheres. Os achados deste estudo observacional parecem representar melhor a população de pacientes com insuficiência cardíaca atendidos e internados nos hospitais por causa de sua cardiopatia.

Semelhante a estudos prévios ${ }^{14,16}$, a doença arterial coronariana foi a causa principal de insuficiência cardíaca na presente amostra, seguida pela cardiopatia hipertensiva. A doença de Chagas, embora relatada como a etiologia primária da insuficiência cardíaca em 1/3 dos casos no Brasil ${ }^{19}$, foi responsável por apenas $10 \%$ dos casos neste estudo. Isto, por sua vez, é concordante com os achados de Barreto et $\mathrm{al}^{20}$, que estudaram transversalmente pacientes com insuficiência cardíaca admitidos no Instituto do Coração (INCOR), em São Paulo. A prevalência da miocardiopatia chagásica foi de apenas $6 \%$. É interessante notar que, na atualidade, em duas das maiores cidades do Brasil (São Paulo e Salvador), a doença de Chagas não parece exercer um papel principal na etiologia da insuficiência cardíaca, provavelmente retratando as mudanças epidemiológicas que ocorreram em nosso país nas últimas décadas, em especial o controle da transmissão vetorial da infecção.

Comorbidades foram freqüentes nesta amostra. Hipertensão arterial sistêmica esteve presente em quase $80 \%$ dos casos. Diabetes mellitus foi observada em 38\% e $15 \%$ dos pacientes tinham história anterior de AVE. Doenças crônicas são mais freqüentes em pacientes mais idosos ${ }^{21,22}$, tal como é o caso desta amostra. Da mesma forma, hipertensão e diabete fazem parte das etiologias de insuficiência cardíaca com função sistólica preservada, especialmente em mulheres idosas ${ }^{17,23,24}$, características que também preenchem o perfil da população aqui avaliada.

A prevalência de diabete é maior em pacientes com insuficiência cardíaca isquêmica do que naqueles de etiologia não isquêmica ${ }^{9}$. No presente estudo, houve uma associação positiva entre diabetes mellitus e etiologia isquêmica. Esta associação foi mais forte nos pacientes $<60$ anos, o que talvez parcialmente explique a presença de doença cardíaca tão grave num grupo de pacientes mais jovens. A prevalência de fibrilação atrial, maior no sexo masculino, foi compatível com achados de outros trabalhos ${ }^{14,20}$ e provavelmente reflita a presença de a idade avançada e a maior gravidade da cardiopatia nesta amostra. Alargamento do QRS (>120mseg) foi muito freqüente e ocorreu em quase metade dos pacientes. Resultados similares já foram descritos ${ }^{25}$, provavelmente relacionados com fração de ejeção ventricular esquerda reduzida. No presente estudo, fração de ejeção $<30 \%$ foi registrada em $44 \%$ dos pacientes, fato que justifica duração prolongada do complexo QRS, pois quanto mais grave a disfunção miocárdica, mais lenta a ativação ventricular.

Alterações na função diastólica foram encontradas em $90 \%$ dos 248 pacientes que realizaram avaliação para esse fim. Destes, $21 \%$ apresentavam função sistólica normal. Este achado é interessante e diferente dos resultados de estudos prévios que quase sempre associam insuficiência cardíaca avançada com disfunção sistólica grave ${ }^{26,27}$. A definição primariamente clínica para insuficiência cardíaca avançada adotada neste trabalho, bem como a elevada freqüência de mulheres nesta amostra, podem justificar esse achado. De fato, há evidências de que a gravidade da manifestação clínica da insuficiência cardíaca não está necessariamente relacionada ao grau de disfunção sistólica ${ }^{28}$.

Anemia, hiponatremia, elevação dos níveis séricos de uréia e creatinina foram achados freqüentes na admissão hospitalar. Esses fatores são reconhecidos atualmente como preditores de mau prognóstico em longo prazo em pacientes com insuficiência cardíaca grave ${ }^{29-31}$. No entanto, o papel prognóstico em curto prazo dessas variáveis não foi ainda estabelecido.

Incidência de desfechos hospitalares - A letalidade hospitalar foi $17,4 \%$. Este valor está de acordo com os resultados de outros estudos envolvendo pacientes admitidos por insuficiência cardíaca ${ }^{20,32-35}$, nos quais a letalidade varia de $6,4 \%^{32}$ a $23,4 \%{ }^{33}$. No Brasil, a letalidade hospitalar para insuficiência cardíaca em instituições da rede pública de saúde é $7,5 \%{ }^{3}$. Embora a presente amostra derive de um hospital de referência da rede privada, onde todos os pacientes têm assistência cardiológica específica e, provavelmente, uma estratégia atual e adequada de tratamento para a doença, estes pacientes eram portadores de insuficiência cardíaca grave e, por causa disso, necessitaram de internação inicial em unidade intensiva.

Esse pode ser um motivo para explicar a letalidade hospitalar mais elevada neste estudo. Entretanto, sendo a insuficiência cardíaca uma doença de diagnóstico clínico, erros de classificação da doença, em ambos os sentidos (dar o diagnóstico em quem não tem a doença ou dar outro diagnóstico em que tem insuficiência cardíaca) são prováveis de ocorrer mais freqüentemente em hospitais 
gerais e da rede pública, o que não é o caso da instituição em questão. É possível que, no presente estudo, a freqüência de erro diagnóstico seja menor, o que fortalece, então, a confiabilidade dos valores encontrados.

Entre os demais desfechos hospitalares observados, chama atenção a elevada incidência de insuficiência renal aguda (42\%) e de embolia pulmonar (15\%). A gravidade da cardiopatia e a grande proporção de pacientes idosos neste estudo podem justificar esses achados.

Análise multivariada para fatores de risco de letalidade hospitalar - No presente estudo, os preditores independentes, registrados na admissão, para letalidade hospitalar foram: história prévia de AVE, fibrilação atrial, idade $>70$ anos, hiponatremia e níveis elevados de creatinina. Embora o papel prognóstico de todas essas características já tenha sido reconhecido para desfechos em longo prazo em insuficiência cardíaca9,29-31,36,37, a descrição do risco potencial para aumentar a letalidade precoce, ainda na fase hospitalar, é um resultado original.

História prévia de AVE e fibrilação atrial na admissão foram associados com redução de sobrevida tardia em pacientes que tinham recebido alta após a primeira internação por insuficiência cardíaca ${ }^{37}$. Outros estudos têm mostrado resultados controversos quanto ao papel da fibrilação atrial nos pacientes com insuficiência cardíaca. Em alguns deles, a presença de fibrilação atrial na admissão não foi associada independentemente com maior risco de mortalidade ${ }^{38-40}$ ou morbidade ${ }^{38-41}$ após a alta hospitalar em pacientes com insuficiência cardíaca avançada. Em análise retrospectiva do estudo SOLVD ${ }^{42}$, envolvendo pacientes sintomáticos e assintomáticos com disfunção sistólica grave do ventrículo esquerdo, os autores descreveram um significante e independente aumento no risco de morte por progressão da insuficiência cardíaca e da morte total no subgrupo com fibrilação atrial na admissão.

Hiponatremia grave e disfunção renal têm sido reconhecidas como alguns dos principais marcadores prognósticos de sobrevida em longo prazo nos pacientes com insuficiência cardíaca ${ }^{43}$. Essas duas alterações metabólicas podem resultar da gravidade da cardiopatia, como também, uma vez presentes em pacientes criticamente doentes, agravar as manifestações da insuficiência cardíaca. De qualquer sorte, o presente estudo levanta a possibilidade de que essas características laboratoriais possam ajudar a identificar, já na admissão hospitalar, um subgrupo de pacientes com risco ainda maior de letalidade precoce.

O papel da idade no prognóstico da insuficiência cardíaca foi avaliado em vários trabalhos. No estudo EPICAL ${ }^{9}$, indivíduos entre 70-80 anos apresentaram um risco independente de morte $50 \%$ maior do que aqueles com idade $<70$ anos. No presente trabalho, os pacientes mais idosos também mantiveram um risco maior de letalidade hospitalar após análise multivariada com nível de significância estatística limítrofe, pois o intervalo de confiança de $95 \%$ desta associação incluiu a unidade.
Isso pode ser um problema relacionado com tamanho da amostra, porém existem outros estudos em pacientes com insuficiência cardíaca grave que não demonstraram que a idade avançada fosse um fator de risco independente para mortalidade ${ }^{4,5,44-46}$

Em estudos prévios, anemia tem sido associada à menor sobrevida tardia em pacientes com disfunção sistólica grave do ventrículo esquerdo (fração de ejeção <35\%) e classe funcional da New York Heart Association I a IV ${ }^{29-31}$. Da mesma forma, a presença de anemia, mesmo em graus relativamente leves, foi associada com piora de sintomas e taxas de readmissão mais elevadas em pacientes com insuficiência cardíaca moderada a grave ${ }^{29}$. No presente estudo não se foi capaz de demonstrar, do ponto de vista estatístico, um risco independente da anemia, mesmo em nível mais importante, na redução da sobrevida hospitalar de pacientes com insuficiência cardíaca avançada. É possível que neste estudo não se tenha tido poder suficiente para detectar essa associação.

$\mathrm{O}$ fato de se terem assumido pacientes com níveis mais leves de anemia no grupo de pacientes não anêmicos (se se considerasse o critério da OMS) pode ter contribuído para isso. Fatores clínicos importantes geralmente associados com pior prognóstico em insuficiência cardíaca, como fração de ejeção do ventrículo esquerdo reduzida e etiologia isquêmica, não foram significantemente associados com morte neste estudo, mesmo na análise bivariada. 0 fato de se estar medindo um desfecho muito precoce em uma amostra de pacientes com doença grave pode justificar a ausência dessas associações. Diferenças no papel prognóstico de variáveis em insuficiência cardíaca já são reconhecidas. A fração de ejeção do ventrículo esquerdo, por exemplo, está associada com menor sobrevida tardia em pacientes portadores de formas leves a moderadas de insuficiência cardíaca, mas não no subgrupo com doença grave $e^{4,5}$. Em relação à etiologia isquêmica, as informações disponíveis ainda são conflitantes. Pacientes com insuficiência cardíaca isquêmica têm sido associados a um prognóstico pior ${ }^{9,47-50}$, comparável $^{51}$ ou mesmo melhor ${ }^{14}$ do que os pacientes com insuficiência cardíaca de etiologia não isquêmica.

\section{Conclusão}

Nesta amostra de pacientes com insuficiência cardíaca, internados em unidade de terapia intensiva, características da admissão foram capazes de predizer de modo independente maior letalidade hospitalar: fibrilação atrial, hiponatremia grave, passado de AVE, idade avançada e disfunção renal. Pacientes com insuficiência cardíaca avançada (estágios $C$ e D ${ }^{52,53}$ ), não inclusos em programas para transplante cardíaco, merecem uma atenção especial em relação àqueles que são atendidos ambulatorialmente com insuficiência cardíaca, pois seu perfil clínico e fatores prognósticos podem apresentar peculiaridades que não foram observadas em estudos prévios. 


\section{REFERÊNCIAS}

1. Massie VM, Shah NB. Evolving trends in the epidemiologic factors of heart failure: rationale for preventive strategies and comprehensive disease management. Am Heart J. 1997;133:703-12.

2. Tecce MA, Pennington JA, Segal BL, Jessup ML. Heart failure: clinical implications of systolic and diastolic dysfunction. Geriatrics. 1999;54:24-33.

3. DATASUS 2004. Ministério da Saúde-SIH/SUS (www.datasus.gov.br)

4. Stevenson WG, Stevenson LW, Middlekauff HR, et al. Improving survival for patients with advanced heart failure: a study of 737 consecutive patients. J Am Coll Cardiol. 1995;26:1417-23.

5. Saxon L, Stevenson WG, Middlekauff, et al. Predicting death from progressive heart failure secondary to ischemic or idiopathic dilated cardiomyopathy. Am J Cardiol. 1993;72:62-5.

6. The SOLVD Investigators. Effect of enalapril on survival in patients with reduced left-ventricular ejection fractions and congestive heart failure. N Engl J Med. 1991;325:293-302.

7. The Task Force for the Working Group on Heart Failure of the European Society of Cardiology. Guidelines for the diagnosis and assessment of heart failure. Eur Heart J. 1995;16:741-51.

8. Rickham PP. Human experimentation. Code of ethics of the world medical association. Declaration of Helsinki. Br Med J. 1964;5402:177.

9. Zannad F, Briancon S, Juilliere Y, et al. Incidence, clinical and etiologic features and outcomes of advanced chronic heart failure: the EPICAL study. J Am Coll Cardiol. 1999;33:734-42.

10. Freitas HFG, Chizzola PR, Paes AT, Lima ACP, Mansur AJ. Risk stratification in a Brazilian hospital-based cohort of 1220 outpatients with heart failure: role of Chagas' heart disease. Int J Cardiol. 2005;102:239-47.

11. Zile MR, Brutsaert DL. New concepts in diastolic dysfunction and diastolic heart failure: Part I. Circulation. 2002;1387-93.

12. DeMaeyer $\mathrm{E}$, Adiels-Yagman $\mathrm{M}$. The prevalence of anaemia in the world. World Health Stat Q. 1985;38:302-16.

13. Campana C, Gavazzi A, Berzuini C, et al. Predictors of prognosis in patients awaiting heart transplantation. J Heart Lung Transplant. 1993;12:756-65.

14. Opasich C, Tavazzi L, Lucci D, et al. Comparison of one-year outcome in women versus men with chronic congestive heart failure. Am J Cardiol. 2000;86:353-7.

15. Kannel WB, Belanger AJ. Epidemiology of heart failure. Am Heart J. $1991 ; 121: 951-7$

16. Samuel RS, Hausdorff JM, Wei JY. Congestive heart failure with preserved systolic function: is it a women's disease? Women's Health Issues. 1999;9:219-22.

17. Ho KK, Pinsky JL, Kannel WB, Levy D. The epidemiology of heart failure: the Framingham study. J Am Coll Cardiol. 1993;22:6A-13A.

18. Lindenfeld J, Krause-Steinrauf H, Salerno J. Where are all the women with heart failure? J Am Coll Cardiol. 1997;30:1417-19.

19. Mady C. Heart failure. Natural history and prognosis. Arq Bras Cardiol. 1994;63:515-7.

20. Barreto ACP, Nobre MRC, Wajngarten M, Canesin MF, Ballas D, SerroAzul JB. Heart failure in a large tertiary hospital of São Paulo. Arq Bras Cardiol. 1998;71:15-20.

21. De Luca L, Gheorghiade M. Hospitalization for worsening chronic heart failure. Ital Heart J. 2004;5(Suppl 6):55S-62S

22. Havranek EP, Masoudi FA, Westfall KA, Wolfe P, Ordin DL, Krumholz HM. Spectrum of heart failure in older patients: results from the
National Heart failure project. Am Heart J. 2002;143:412-7.

23. Krumholz HM, Parent EM, Tu N, et al. Readmission after hospitalization for congestive heart failure among Medicare beneficiaries. Arch Intern Med. 1997;157:99-104.

24. Levy D, Larson MG, Vasan RS, Kannel WB, Ho KLK. The progression from hypertension to heart failure. J Am Coll Cardiol. 1996;275:1557-62.

25. Iuliano S, Fisher SG, Karasik PE, Fletcher RD, Singh SN, for the Department of Veterans Affairs Survival Trial of Antiarrhythmic Therapy in Congestive Heart failure. QRS duration and mortality in patients with congestive heart failure. Am. Heart J. 2002; 143:1085-91.

26. Wheeldon NM, Clarkson P, MacDonald TM. Diastolic heart failure. Eur Heart J. 1994;15:1689-97.

27. Vasan RS, Benjamin EJ, Levy D. Prevalence, clinical features and prognosis of diastolic heart failure: an epidemiologic perspective. J Am Coll Cardiol. 1995;26:1565-74.

28. Gandhi SK, Powers JC, Nomeir AM, et al. The pathogenesis of acute pulmonary edema associated with hypertension. N Engl J Med. 2001;344:17-22.

29. Horwish TB, Fonarow GC, Hamilton MA, MacLellan WR, Borenstein $\mathrm{J}$. Anemia is associated with worse symptoms, greater impairment in functional capacity and a significant increase in mortality in patients with advanced heart failure. J Am Coll Cardiol. 2002; 11:1780-6.

30. Al-Ahmad A, Rand WM, Manjunath G, et al. Reduced kidney function and anemia as risk factors for mortality in patients with left ventricular dysfunction. J Am Coll Cardiol. 2001; 38:955-62.

31. Mozaffarian D, Nye R, Levy WC. Anemia predicts mortality in severe heart failure. J Am Coll Cardiol. 2003;41:1933-9.

32. Polanczyk CA, Rohde LE, Philbin EA, Di Salvo TG. A new casemix adjustment index for hospital mortality among patients with congestive heart failure. Med Care. 1998;36:1489-99.

33. Stewart S, Demers C, Murdoch DR, et al. Substantial between-hospital variation in outcome following first emergency admission for heart failure. Eur. Heart J. 2002;23:650-7.

34. Lee DS, Johansen H, Gong Y, Hall RE, Tu JV, Cox JL. Canadian Cardiovascular Outcomes Research Team. Regional outcomes of heart failure in Canada. Can J Cardiol. 2004; 20:599-607.

35. Tavares LR, Victer H, Linhares JM, et al. Epidemiologia da insuficiência cardíaca descompensada em Niterói - Projeto EPICA - Niterói Arq Bras Cardiol. 2004;82:121-4.

36. Gottdiener JS, Mcclelland RL, Marshall R, et al. Outcome of congestive heart failure in elderly persons: influence of left ventricular systolic function. The Cardiovascular Health Study. Ann Intern Med. 2002;137:631-9.

37. Cleland JGF, Gemmell I, Khand A, Boddy A. Is the prognosis of heart failure improving? Eur J Heart Fail. 1999;1:229-41.

38. Swedberg K, Olsson LG, Charlesworth A, et al. Prognostic relevance of atrial fibrillation in patients with chronic heart failure on long-term treatment with beta-blockers: results from COMET. Eur Heart J. 2005; accessed in http://eurheartj.oupjournals.org/cgi/rapidpdf/ehi166v1.

39. Carson PE, Johnson GR, Dunkman WB, Fletcher RD, Farrell L, Cohn $\mathrm{JN}$. The influence of atrial fibrillation on prognosis in mild to moderate heart failure. The V-Heft Studies. The V-Heft VA Cooperative Studies Group. Circulation. 1993;87:VI102-10.

40. Crijns HJ, Tjeerdsma G, de Kam PJ, et al. Prognostic value of the presence and development of atrial fibrillation in patients with advanced chronic heart failure. Eur Heart J. 2000;21:1238-45.

41. Ahmed A, Thornton P, Perry GJ, Allman RM, DeLong JF. Impact of atrial fibrillation on mortality and readmission in older adults hospitalized 
with heart failure. Eur J Heart Fail. 2004;6:421-6.

42. Dries DL, Exner DV, Gersh BJ, Domanski MJ, Waclawiw MA, Stevenson LW. Atrial fibrillation in associated with an increased risk for mortality and heart failure progression in patients with asymptomatic and symptomatic left ventricular systolic dysfunction: a retrospective analysis of the SOLVD trials. J Am Coll Cardiol. 1998;32:695-703.

43. Aaronson KD, Schwartz JS, Chen TM, Wong KL, Goin JE, Mancini $\mathrm{DM}$. Development and prospective validation of a clinical index to predict survival in ambulatory patients referred for cardiac transplant evaluation. Circulation. 1997;95:2660-7.

44. Anguita M, Arizon JM, Bueno G, et al. Clinical and hemodynamic predictors of survival in patients aged $<65$ years with severe congestive heart failure secondary to ischemic or nonischemic dilated cardiomyopathy. Am J Cardiol .1993;72:413-7.

45. Brophy JM Deslauriers G, Rouleau JL. Long term prognosis of patients presenting to the emergency room with descompensated congestive heart failure. Can J Cardiol. 1994;10:543-47.

46. Adams KF, Dunlap SH, Sueta CA, et al. Relation between gender, etiology and survival in patients with symptomatic heart failure. J Am Coll Cardiol. 1996;28:1781-8.

47. Bourassa MG, Gurné O, Bangdiwala SI, et al. Natural history and patterns of current practice in heart failure. J Am Coll Cardiol. 1993;22:14A-19A.
48. Packer M, O'Connor CM, Ghali JK, et al. Effect of amlodipine on morbidity and mortality in severe chronic heart failure. N Engl J Med. 1996;335:1107-14.

49. Franciosa JA, Wilen M, Zietsche S, Cohn JN. Survival in men with severe chronic left ventricular failure due to either coronary heart disease or idiopatic dilated cardiomyopathy. Am J Cardiol. 1983;51:831-6.

50. Cohn JN, Rector TS. Prognosis of congestive heart failure and predictors of mortality. Am J Cardiol. 1988;62:25A-30A.

51. Wilson JR, Schwartz JS, Sutton MS. Prognosis in severe heart failure: relation to hemodynamic measurements and ventricular ectopic activity. J Am Coll Cardiol. 1983;2:403-10.

52. Butler J, Khadim G, Paul KM, et al. Selection of patients for heart transplantation in the current era of heart failure therapy. J Am Coll Cardiol .2004; 43:787-93.

53. Hunt SA, Abraham WT, Chin MH, et al. ACC/AHA 2005 guideline update for the diagnosis and management of chronic heart failure in the adult: a report of the American College of Cardiology/American Heart Association Task Force on Practice Guidelines (Writing Committee to Update the 2001 Guidelines for the Evaluation and Management of Heart Failure). American College of Cardiology Web Site. Available at: http://www.acc.org/clinical/guidelines/failure/index.pdf. 